\title{
Analyzing the clinical significance of postoperative methotrexate in the management of early abdominal pregnancy: analysis of 10 cases
}

\author{
Emmanuel Ansong, Gaby Sukma Illahi, Laien Shen, Xueqing Wu \\ The First Affiliated Hospital of Wenzhou Medical University, China
}

\begin{abstract}
Objectives: To assess the clinical value and treatment outcomes of postoperative methotrexate (MTX) therapy in the management of early abdominal pregnancy.

Material and methods: We retrospectively analyzed ten (10) cases of early abdominal pregnancy at our hospital between 7th August, 2006 and 20th April, 2017.

Results: Out of the ten (10) cases identified, six (6) patients and four (4) patients underwent surgery (laparotomy or laparoscopy) only and surgery (laparotomy or laparoscopy) plus IM $50 \mathrm{mg} / \mathrm{m}^{2}$ methotrexate (MTX) within 24 hours of surgery respectively. The gestation age and serum $\beta$-HcG levels were significantly lower $(p<0.05,6.0 \pm 1.82$ and $8073.2 \pm 9561.0)$ in the surgery plus MTX group in comparison to $(7.33 \pm 3.61$ and $15625 \pm 21275.2)$ for the surgery only group. Ultrasound imaging findings reported extra uterine pregnancy in all cases and diagnostic surgery was necessary to locate precise site of implantation to plan further treatment. Days of hospitalization were shorter in the surgery + MTX group than in the surgery only group $(3.00 \pm 0.816$ versus $5.66 \pm 2.80)$.

Conclusions: Earliness in diagnosis coupled with the appropriate (methotrexate) MTX regime could help prevent unwanted complications that could arise from delayed or misdiagnosis.
\end{abstract}

Key words: abdominal ectopic pregnancy; early abdominal pregnancy; serum $\beta-\mathrm{HcG}$; methotrexate; diagnostic laparoscopy

Ginekologia Polska 2019; 90, 8: 438-443

\section{INTRODUCTION}

Abdominal ectopic pregnancies (AEP) are the rarest form ectopic pregnancies. With an estimated incidence of $1 / 6000$ to $1 / 9000$ births and $1 / 2200$ to $1 / 10200$ pregnancies, their mortality rate is reported to be 7.7 times and 89.8 times higher in tubal pregnancy and intrauterine pregnancy respectively [1,2]. They can be referred to as; early when gestation is at or before 20 weeks and late when gestation is after 20 weeks [3]. Although rarely used clinically, signs and symptoms, levels of serum beta human chorionic gonadotrophin (ß-hCG), ultrasound and magnetic resonance imaging can be diagnostically productive in certain instances [4,5]. Diagnostic laparoscopy has become the cornerstone treatment for AEP, but they can also be managed medically with systemic or local methotrexate (MTX), ultrasound guided potassium chloride (KCL), danazol and mifepristone $[6,7]$. While few cases of early abdominal cases have been managed with surgery plus methotrexate, no studies, according to our knowledge have investigated the importance of methotrexate in these scenarios [8, 9]. We retrospectively studied the records of ten (10) who were diagnosed with early abdominal pregnancy in our hospital and were managed with surgery alone or surgery plus MTX. This study was necessary because, an in-depth understanding of the presentation, diagnosis and management of this rare form of ectopic pregnancy would help to minimize cases of late or misdiagnosis which could result in loss of fertility or even maternal mortality [10].

\section{MATERIAL AND METHODS}

A single institution retrospective study was carried out between $7^{\text {th }}$ August, 2006 and $20^{\text {th }}$ April, 2017 at the First Affiliated Hospital of Wenzhou Medical University, Zhejiang Province, China where ten (10) cases of early abdominal pregnancies were identified. Information on patient's biodata, pregnancy history, presenting features and reproductive outcomes were retrieved from our computerized medical chart as documented by three different surgeons who oversaw their 
management. Although high titers of serum ß-HCG prompted the suspicion of ectopic pregnancy, the definitive diagnosis of early abdominal pregnancy (before 20 weeks gestation) was made based on perioperative findings at varying implantation sites and was later confirmed by pathological examination. Cases of tubal pregnancies and pregnancies at locations that do not fit the criteria of early abdominal pregnancy and pregnancy beyond 20 weeks gestation were all excluded. Permission to carry on with this study was granted after careful evaluation by the Ethics Committee of The First Affiliated Hospital of Wenzhou Medical University (wydw2017-0115) and after patients gave their verbal consent through telephone calls. We subsequently divided the 10 cases into two main categories depending on the treatment modalities each patient received as surgery only and surgery plus IM $50 \mathrm{mg} / \mathrm{m}^{2}$ methotrexate (MTX). Details on maternal age, pregnancy history, gestational age, presenting symptoms, pre and post-operative blood levels of beta human chorionic gonadotropin (B-hCG), implantation sites, first and second lines of treatment, blood loss, days of hospitalization and reproductive outcomes were all documented. Amount of blood loss during laparoscopy was evaluated from the suction-irrigation tube and from direct suction combined with the weight of soaked pads during laparotomy. Presenting symptoms among the patients included abdominal pain and vaginal bleeding. Statistical calculations were done using independent sample t-test, where significant differences between the two groups were deemed at $\mathrm{p}<0.05$.

\section{RESULTS}

\section{Clinical characteristics of the participants}

The comprehensive information on all the ten (10) cases of primary abdominal pregnancies is shown in Table 1.
The mean \pm SD for age was $29.9 \pm 5.93$ years while the gestational age as estimation by last menstrual period (LMP) was $6.8 \pm 2.973$ weeks. The median gravidity and parity was 2 (range 1-3) and 1 (range 1-3) respectively. Risk factors recorded include previous pelvic inflammatory disease (2 of 10, 20\%), history of In Vitro Fertilization (3 of 10, 30\%), previous tubal surgery ( 3 of $10,30 \%$ ) and two ( 2 of 10 , $20 \%)$ patients with no existing factors. In all the 10 cases, 4 patients presented with complaints of vaginal bleeding and abdominal pain, 5 with only abdominal pain and only one with vaginal bleeding. Case 8 reported with the highest level of serum B-hCG of 58121 lu/L. Details of the report on preoperative ultrasonographic findings are summarized in Table 2. Only three cases were managed with laparotomy, the remaining seven cases (2010-2017) were all managed laparoscopically. Varied sites of implantation reported include pouch of Douglas (3 of 10,30\%), mesosalpinx (3 of $10,30 \%)$, vesicouterine pouch ( 2 of $10,20 \%$ ), intestinal wall ( 1 of $10,10 \%$ ) and omentum ( 1 of 10, $10 \%$ ). Only 4 patients (Case $5,6,7,9)$ received a second line treatment of IM $50 \mathrm{mg} / \mathrm{m}^{2}$ methotrexate (MTX) within 24-hours of surgery. The overall mean \pm SD for days of hospitalization was $4.6 \pm 2.547$ days with Case 1 staying the longest ( 11 days). Four patients were lost to follow up, but two (case 5 and 6) out of the six patients we contacted in 2017 had achieved spontaneous delivery at 4 and 2 years respectively following their treatment.

\section{Pre and post-operative serial measurement of serum B-hCG}

Graphical representation of serum B-hCG levels on the first day of admission (Day 0 ) and throughout the course

\begin{tabular}{|c|c|c|c|c|c|c|c|c|c|c|c|c|}
\hline \multirow{2}{*}{ ঠ் } & \multirow[b]{2}{*}{ ঙ্ঠ } & \multirow[b]{2}{*}{ ğ } & \multirow{2}{*}{ 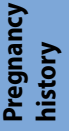 } & \multirow{2}{*}{$\begin{array}{l}\text { Risk } \\
\text { factors }\end{array}$} & \multirow{2}{*}{$\begin{array}{l}\text { Weeks } \\
\text { of GA }\end{array}$} & \multirow{2}{*}{ Symptoms } & \multirow{2}{*}{$\begin{array}{l}\text { Serum } \beta \text {-HCG } \\
\text { level (lu/L) }\end{array}$} & \multirow{2}{*}{$\begin{array}{l}\text { Implantation } \\
\text { site }\end{array}$} & \multicolumn{2}{|c|}{ Treatment } & \multirow{2}{*}{$\begin{array}{l}\text { Days In } \\
\text { hospital }\end{array}$} & \multirow{2}{*}{$\begin{array}{l}\text { Reproductive } \\
\text { outcome }\end{array}$} \\
\hline & & & & & & & & & $1^{\text {st }} \mathrm{TX}$ & $2^{\text {nd }} T X$ & & \\
\hline 1 & 2006 & 33 & G2P0 & Previous TS & 13 & AP & 6,414 & Mesosalpinx & LAP & - & 11 & No delivery \\
\hline 2 & 2006 & 32 & G3P2 & Previous TS & 7 & $\mathrm{VB}+\mathrm{AP}$ & 11,705 & POD & LAP & - & 5 & Post TL \\
\hline 3 & 2008 & 27 & G1P1 & PID & 5 & $V B+A P$ & 12,048 & Mesosalpinx & LAP & - & 3 & - \\
\hline 4 & 2010 & 32 & G2P1 & None & 6 & VB & 307 & Intestinal wall & LSC & - & 4 & - \\
\hline 5 & 2011 & 25 & G1P1 & PID & 7 & $A P$ & 20,731 & POD & LSC & MTX & 3 & $1 \mathrm{SD}$, after 4 years \\
\hline 6 & 2013 & 27 & G2P1 & None & 8 & $\mathrm{VB}+\mathrm{AP}$ & 512 & VUP & LSC & MTX & 4 & $1 \mathrm{SD}$, after 2 years \\
\hline 7 & 2014 & 18 & G1P0 & IVF & 5 & $V B+A P$ & 842 & Mesosalpinx & LSC & MTX & 3 & - \\
\hline 8 & 2015 & 37 & G2P1 & Previous TS & 10 & $A P$ & 58,121 & POD & LSC & - & 6 & - \\
\hline 9 & 2017 & 30 & GOPO & IVF & 4 & $A P$ & 10,208 & Omentum & LSC & MTX & 2 & Infertility History \\
\hline 10 & 2017 & 38 & GOPO & IVF & 3 & AP & 5,155 & VUP & LSC & _ & 5 & Infertility History \\
\hline
\end{tabular}

AP - abdominal pain; G - gravidity; GA — gestational age; IVF — in vitro fertilization; LAP — laparotomy; LSC — laparoscopy; MTX — methotrexate; P - parity; PID — pelvic inflammatory disease; POD — pouch of Douglas; SD — spontaneous delivery; $T L$ - tubal ligation; $T S$ - tubal surgery; TX — treatment; $V B$ - vaginal bleeding; VUP — vesico-uterine pouch 


\begin{tabular}{|l|l|l|}
\hline Table 2. Transvaginal ultrasound imaging characteristics \\
\hline Case & Radiologic comments \\
\hline 1 & A cystic mass shadow measuring $16 \times 10 \times 13 \mathrm{~mm}$ in the posterior wall of uterus + pelvic fluid \\
\hline 2 & Gestation sac consistent with 6 weeks in the pouch of Douglas + free peritoneal fluid \\
\hline 3 & A heterogenous mass $29 \times 19 \times 18 \mathrm{~mm}$ near the left ovary + suspected bleeding in the pouch of Douglas \\
\hline 4 & A $21 \times 29 \times 42 \mathrm{~mm}$ cystic area + pelvic fluid with internal echoes \\
\hline 5 & Free fluid in the lower abdomen + pelvic solid mass in the pouch of Douglas \\
\hline 6 & A $22 \times 17 \mathrm{~mm}$ vital embryo + cardiac activity in the pouch of Douglas \\
\hline 7 & A possible extra-uterine (right side of the uterus) with an empty uterus and normal adnexa \\
\hline 8 & A hyperechogenic mass $(20 \times 27 \times 23 \mathrm{~mm})$ behind the uterus on the left side + pelvic fluid \\
\hline 9 & No intrauterine gestational sac + possible hemantoma $(43 \times 28 \times 31 \mathrm{~mm})$ close to the left ovary \\
\hline 10 & Mass-like area measuring $27 \times 36 \times 34 \mathrm{~mm}+$ fluid in the anterior and posterior cul-de-sacs \\
\hline
\end{tabular}

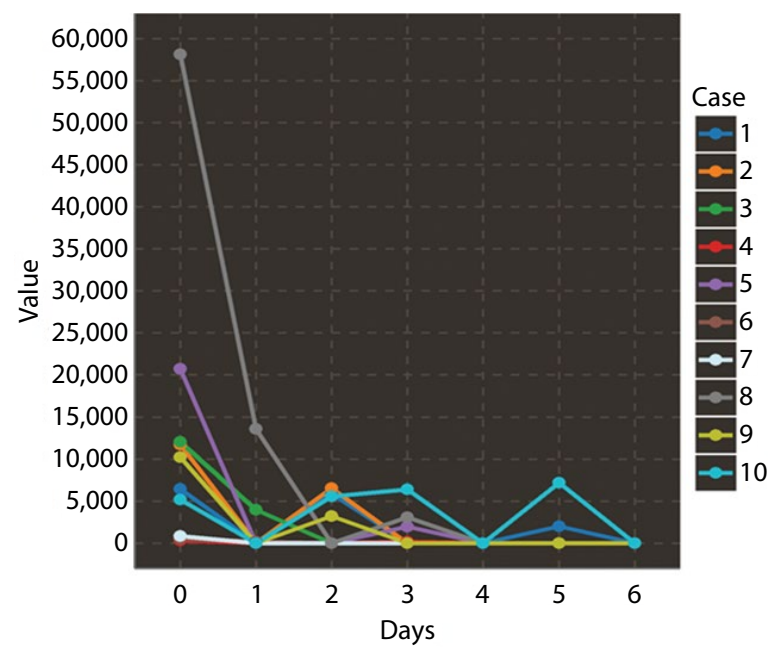

Figure 1. Serial measurement of serum ß-hCG. Day 0 represents the first day of admission and days $1,2,3,4,5$ and 6 represent postoperative days

of treatment (Post-operative Day 1-6) is shown in Figure 1. Case 8 and Case 4 recorded the highest ( $58121 \mathrm{lu} / \mathrm{L}$ ) and the lowest (307 lu/L) levels of serum B-hCG respectively. Levels of serum B-hCG were also assessed on $1^{\text {st }}, 2^{\text {nd }}, 3^{\text {rd }}$, $4^{\text {th }}, 5^{\text {th }}$ and $6^{\text {th }}$ post-operative days. All patients displayed a sharp decline in their levels on the first day after surgery. However cases 2, 9, 10 showed a slight upsurge in their levels on post-operative day 2 but not as high as the levels on the day of admission (Day 0). Serum ß-hCG levels continued to plummet from post-operative day 3 through to $6^{\text {th }}$ operative day except for case 10 that showed an obvious fluctuating trend throughout the course of treatment.

\section{Correlation between treatment modalities and clinical parameters}

The surgery plus methotrexate group presented with a mean gestational age of $6.0 \pm 1.82$ vs the $7.33 \pm 3.61$ among the surgery only group with a significant difference

\begin{tabular}{|l|l|l|}
\hline \multicolumn{3}{|l}{ Table 3: Management of primary abdominal pregnancy: surgery } \\
only vs surgery plus methotrexate (MTX)
\end{tabular}

$(p=0.000049)$. The surgery only group recorded more number of abortions than the surgery plus methotrexate group. Levels of serum B-hCG on the day of admission were significantly correlated and higher in the surgery only group that $(15625 \pm 21275.2$ vs $8073.2 \pm 9561.00$, $p=0.046065$. Further successive measurements of serum B-hCG throughout the course of therapy and on the day of discharge demonstrated that the group that was managed with surgery and methotrexate had lower levels of serum B-hCG in comparison to the group that had undergone surgery only $(1071.4 \pm 1269.11$ vs $5935.66 \pm 4378.6$, $p=0.01824)$. The two groups were significantly correlated in terms of length of hospital stay $(p<0.05)$. The surgery plus methotrexate group were discharged from hospital much earlier than the group who received surgery only $(3.00 \pm 0.816$ vs $5.66 \pm 2.80)$ Table 3 .

\section{DISCUSSION}

From a select population of 8,547 ectopic pregnancies diagnosed in our institution between $7^{\text {th }}$ August, 2006 and $20^{\text {th }}$ April, 2017, only $10(0.11 \%)$ cases were diagnosed and treated as early abdominal pregnancy representing the least form of ectopic pregnancy in our institution. Endo- 
metriosis, the current usage of an intrauterine device and a history of tubal surgery or ectopic pregnancy are some of the documented risk factors for abdominal pregnancy and also implicated in other forms of ectopic pregnancies [11]. The sites of implantation from the commonest to the least reported include the pouch of Douglas (pouches surrounding the uterus), uterine serosa and adnexa, abdominal organs, omentum, bowel/appendix, liver (common site is the right lobe), spleen, retroperitoneal and the wall of the abdomen. Similar implantation locations were discovered in this present study. All cases indexed in this study met the established criteria for primary abdominal pregnancy: (1) normal ovaries and tubes with no evidence of injury, (2) no evidence of uteroplacental fistula, and (3) the pregnancy is adhered exclusively to the peritoneal surface early enough in gestation to eliminate the possibility of secondary implantation after primary nidation in the fallopian tube [12].

The diagnosis of early abdominal pregnancy is a huge clinical hurdle as most of the investigative tools are sometimes unreliable. Presenting symptoms are not strong diagnostic tools due to the absence of typical pathognomonic symptoms to properly define early abdominal pregnancy. Additionally, due to the upsurge of serum B-hCG levels in other forms ectopic pregnancies and the difficulty associated with singling out early abdominal pregnancy based on ultrasonographic features, these investigations are not very instrumental in diagnosis as well $[4,5,13]$. In this present study, diagnostic surgery was the first line option due to the worsening nature of their symptoms and our high suspicion of ectopic rupture. We proceeded quickly with either diagnostic laparotomy or laparoscopy to avert any mortalities or complications and to also aid in the formulation of a better post-operative treatment regime. Until recently, laparotomy was the conventional surgical approach but several reports have exhibited the novelty of diagnostic laparoscopic approach in early abdominal pregnancy management $[9,14]$. In one study to compare the treatment outcomes of these two modalities in abdominal pregnancies, it was observed that, with advancement in technology and improved surgical skills more cases can be confidently managed laparoscopically. The authors also reported varying advantages of management with laparoscopy ranging from reduced blood loss to short hospital stay [15].

Diagnostic laparoscopy was carried out uneventfully and peri-operative findings included normal uterus and adnexa in all cases, hemoperitoneum of about $250 \mathrm{cc}$ and $320 \mathrm{cc}$ for cases 3 and 9 respectively and were transfused with 2 units of whole blood and 3 units of packed red blood cells respectively during surgery. Further examination of the abdomen revealed bloody lesions that were loosely or tenaciously adhered to varying locations in the abdomen. These lesions were removed with the help of non-traumatic laparoscopic forceps and bipolar scissors. In all laparoscopic cases, we achieved hemostasis with the help of bipolar coagulation ruling out the need for suturing. Laparotomy was successfully carried out on three patients (Case 1, 2, 3) but factors that necessitated this choice of management were not recorded. Clinically, the decision to opt for laparotomy can be decided on factors like the anatomical position of the pregnancy or the laparoscopic skills and confidence of the surgeon [16]. Because of the likelihood of implantation on vascularized surfaces which may have high propensity to separate at any time to cause heavy bleeding, the decision between these two management modalities should be precise and prompt [17].

Methotrexate (MTX) has proven to be an effective agent in managing ectopic pregnancy medically since its introduction in 1982 [18]. Its effectiveness has been likened to surgery in terms of treatment results and preservation of future fertility $[19,20]$. Successful treatment which meant avoidance of surgery was recorded to be $94.4 \%$ in women with initial $\beta$-hCG levels of 1000 to $1999 \mathrm{mlU} / \mathrm{mL}$ and $81.8 \%$ in their counterparts with levels from 10,000 to $150,000 \mathrm{mlU} / \mathrm{mL}$. In early abdominal pregnancy, however, there is a high probability of reverting to surgical management among patients who receive systemic or local injections of MTX as first line therapy $[6,13,21]$. The decision for the use of post-operative MTX in ectopic pregnancy include cases were, potential life-threatening torrential bleeding can be foreseen or when there is an upswing or a less than 20\% drop in the levels of serum B-hCG measured consecutively on 3 days apart or prophylactically when incomplete resection or persistent ectopic pregnancy is likely $[22,23]$. By virtue of the position of their lesions on highly vascularized organs and presence of hemoperitoneum which was suggestive of rupture, the surgeons advised the use of methotrexate (MTX) in Case 5,6 and 7. On the other hand, Case 9 received methotrexate (MTX) because the clinicians suspected persistence of her pregnancy which was evidenced by upsurge in her serum HCG levels on post-operative day 2 . Although, cases 2, 8 and 10 witnessed fluctuations in their serum HCG levels post-operatively to also permit the use of MTX, contra-indicatory factors like existing peptic ulcer (Case 2), hemodynamic instability (Case 8) and non-compliance (Case 10) did not make them good candidates [24]. Regarding treatment outcomes, we cannot confidently draw any valid inference from post-operative serum levels among the two groups. The gestational ages and the pre-operative HCG levels were lower in the surgery + MTX group in comparison to the surgery only group and therefore it is likely the reason why their serum B-HCG levels on hospital discharge were lower. Additionally, the reduced blood loss among the surgery + MTX group could also account for their shorter days of hospitalization. Data from this present study regarding future fertility is inconclusive, although the use of MTX is 
reported to enhance future fertility in other forms of tubal ectopic pregnancy [25].

\section{CONCLUSIONS}

The rarity, complex history and atypical clinical characteristics of abdominal ectopic pregnancy make its diagnosis and management extremely puzzling. Conservative surgery and medical therapy can be regarded as appropriate treatment modalities but diagnostic laparoscopy has become the optimal choice because it ensures earliness in diagnosis and offers a better view of the location to determine the size and relative vascularity. A thorough inspection of the abdominal viscera should be carried out to rule out implantation at uncommon sites. Adjuvant MTX therapy can help reduce complications especially when torrential bleeding from vascularized sites can be anticipated but the selection of good candidates still remains crucial. The paucity of information on abdominal pregnancy retrieved from only case reports and series limits this study to a larger extent. Non-compliance from patients in this indexed study makes the data on serial post-operative serum B-HCG levels statistically insufficient to draw any strong conclusions from in terms of treatment outcomes. Further studies are therefore needed to adequately examine the usefulness of post-operative MTX clinically.

\section{Funding}

Shenzhen Science and Technology Innovation Committee Project (Basic Research Program) [No. JCYJ20170818100355168] and Zhejiang Provincial Natural Science Foundation of China [No. LY17H040009]

\section{Acknowledgement}

The authors would like to thank Dr Zhou Kai and Dr Zhu Fangfang from the Gynecology department of First Affiliated Hospital of Wenzhou Medical University for their contributions toward the development of this study.

\section{Statement of ethics}

Permission to carry on with this study was granted after careful evaluation by the Ethics Committee of The First Affiliated Hospital of Wenzhou Medical University (wydw20170115) and after patients gave their verbal consent through telephone calls.

\section{Authors' contributions}

EA conceived, designed the study and drafted the manuscript, GI and LS helped data collection, analyzed the data and help draft the manuscript. XW revised the manuscript, providing intellectual content. All authors commented on and approved the final manuscript.

\section{REFERENCES}

1. Atrash HK, Friede A, Hogue CJ. Abdominal pregnancy in the United States: frequency and maternal mortality. Obstet Gynecol. 1987; 69(3 Pt 1): 333-337, indexed in Pubmed: 3822281.

2. Sunday-Adeoye I, Twomey D, Egwuatu EV, et al. A 30-year review of advanced abdominal pregnancy at the Mater Misericordiae Hospital, Afikpo, southeastern Nigeria (1976-2006). Arch Gynecol Obstet. 2011;283(1): 19-24, doi: 10.1007/s00404-009-1260-4, indexed in Pubmed: 19876640.

3. Martin J, McCaul J. Emergent Management of Abdominal Pregnancy. Clin Obstet Gynecol. 1990; 33(3): 438-447, doi: 10.1097/00003081199009000-00008.

4. Costa SD, Presley J, Bastert G. Advanced abdominal pregnancy. Obstet Gynecol Surv. 1991; 46(8): 515-525, indexed in Pubmed: 1886705.

5. Jurkovic $D$, Mavrelos $D$. Catch me if you scan: ultrasound diagnosis of ectopic pregnancy. Ultrasound Obstet Gynecol. 2007; 30(1): 1-7, doi: 10.1002/uog.4077, indexed in Pubmed: 17587215.

6. Vignali $M$, Busacca $M$, Brigante $C$, et al. Abdominal pregnancy as a result of gamete intrafallopian transfer (GIFT) and subsequent treatment with methotrexate: case report. Int J Fertil. 1990; 35(5): 280-283, indexed in Pubmed: 1980663.

7. Raughley MJ, Frishman GN. Local treatment of ectopic pregnancy. Semin Reprod Med. 2007; 25(2): 99-115, doi: 10.1055/s-2007-970049, indexed in Pubmed: 17377897.

8. Crespo R, Campillos JM, Villacampa A, et al. Successful methotrexate treatment of an abdominal pregnancy in the pouch of Douglas. Clin Exp Obstet Gynecol. 2008; 35(4): 289-290, indexed in Pubmed: 19205447.

9. Koo HS, Bae JuY, Kang IS, et al. Laparoscopic management of early primary peritoneal pregnancy: a case report. Clin Exp Reprod Med. 2011; 38(2): 109-114, doi: 10.5653/cerm.2011.38.2.109, indexed in Pubmed: 22384428.

10. Fylstra DL. Ectopic pregnancy not within the (distal) fallopian tube etiology, diagnosis, and treatment. Am J Obstet Gynecol. 2012; 206(4): 289-299, doi: 10.1016/j.ajog.2011.10.857, indexed in Pubmed: 22177188.

11. Cobellis L, Stradella L, Messalli EM. Contribution to the choice of therapy in abdominal pregnancy. Panminerva Med. 2000; 42(2): 159-161, indexed in Pubmed: 10965779.

12. Poole A, Haas D, Magann EF. Early abdominal ectopic pregnancies: a systematic review of the literature. Gynecol Obstet Invest. 2012; 74(4): 249-260, doi: 10.1159/000342997, indexed in Pubmed: 23108297.

13. Stucki D, Buss J. The ectopic pregnancy, a diagnostic and therapeutic challenge. J Med Life. 2008; 1(1): 40-48, indexed in Pubmed: 20108478.

14. Ginath S, Malinger G, Golan A, et al. Successful laparoscopic treatment of a ruptured primary abdominal pregnancy. Fertil Steril. 2000; 74(3): 601-602, doi: 10.1016/s0015-0282(00)00686-5, indexed in Pubmed: 10973664.

15. Shaw SW, Hsu JJ, Chueh HY, et al. Management of primary abdominal pregnancy: twelve years of experience in a medical centre. Acta Obstet Gynecol Scand. 2007; 86(9): 1058-1062, doi: 10.1080/00016340701434476, indexed in Pubmed: 17712645.

16. delRosario R, el-Roeiy A. Abdominal pregnancy on the bladder wall following embryo transfer with cryopreserved-thawed embryos: a case report. Fertil Steril. 1996; 66(5): 839-841, doi: 10.1016/s00150282(16)58648-8, indexed in Pubmed: 8893697.

17. Shafi SM, Malla MA, Salaam PA, et al. Abdominal pregnancy as a cause of hemoperitoneum. J Emerg Trauma Shock. 2009; 2(3): 196-198, doi 10.4103/0974-2700.55342, indexed in Pubmed: 20009311.

18. Tanaka T, Hayashi H, Kutsuzawa T, et al. Treatment of interstitial ectopic pregnancy with methotrexate: report of a successful case. Fertil Steril. 1982; 37(6): 851-852, doi: 10.1016/s0015-0282(16)46349-1, indexed in Pubmed: 7084507.

19. Hajenius PJ, Engelsbel S, Mol BW, et al. Randomised trial of systemic methotrexate versus laparoscopic salpingostomy in tubal pregnancy. Lancet. 1997; 350(9080): 774-779, doi: 10.1016/s0140-6736(97)05487-1, indexed in Pubmed: 9297998.

20. Alleyassin A, Khademi A, Aghahosseini M, et al. Comparison of success rates in the medical management of ectopic pregnancy with single-dose and multiple-dose administration of methotrexate: a prospective, randomized clinical trial. Fertil Steril. 2006; 85(6): 1661-1666, doi: 10.1016/j. fertnstert.2005.11.055, indexed in Pubmed: 16650421.

21. Giannopoulos T, Katesmark M. Ruptured tubal ectopic pregnancy with secondary implantation in the pouch of Douglas. J Obstet Gynaecol. 2004; 24(2): 199-200, doi: 10.1080/01443610410001643489, indexed in Pubmed: 14766481. 
22. Vermesh M, Silva PD, Sauer MV, et al. Persistent tubal ectopic gestation: patterns of circulating beta-human chorionic gonadotropin and progesterone, and management options. Fertil Steril. 1988; 50(4): 584-588, doi: 10.1016/s0015-0282(16)60187-5, indexed in Pubmed: 2458973.

23. Gracia CR, Brown HA, Barnhart KT. Prophylactic methotrexate after linear salpingostomy: a decision analysis. Fertil Steril. 2001; 76(6): 1191-1195, doi: 10.1016/s0015-0282(01)02906-5, indexed in Pubmed: 11730749.
24. Bachman EA, Barnhart K. Medical management of ectopic pregnancy: a comparison of regimens. Clin Obstet Gynecol. 2012; 55(2): 440-447, doi: 10.1097/GRF.0b013e3182510a73, indexed in Pubmed: 22510626.

25. Dhar H, Hamdi I, Rathi B. Methotrexate treatment of ectopic pregnancy: experience at nizwa hospital with literature review. Oman Med J. 2011; 26(2): 94-98, doi: 10.5001/omj.2011.24, indexed in Pubmed: 22043392. 